\title{
A broad analysis of short-term overreactions in the market for cryptocurrencies
}

\author{
Tobias Kellner, Dominik Maltritz \\ University of Erfurt, Erfurt, Germany \\ Published in Journal of Economic Studies
}

\section{JEL classification:}

G12, G14

\section{Keywords:}

cryptocurrencies, overreaction, market efficiency, Bitcoin, abnormal returns, magnitude effect

\section{Declarations of interest:}

none

\author{
Original published in: Journal of Economic Studies, 2021, Vol. ahead-of-print, No. \\ ahead-of-print. \\ Original published: $\quad$ 2021-12-28 \\ ISSN: $\quad 0144-3585$ \\ DOI: $\quad$ 10.1108/JES-09-2021-0488 \\ Visited: $\quad 01.02 .2022$ \\ Open Access pathway: Accepted Version
}

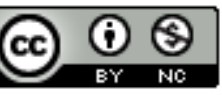

This author accepted manuscript is deposited under a Creative Commons Attribution Non-commercial 4.0 International (CC BYNC) licence. This means that anyone may distribute, adapt, and build upon the work for non-commercial purposes, subject to full attribution. If you wish to use this manuscript for commercial purposes, please contact permissions@emerald.com 


\begin{abstract}
We analyze market inefficiencies in the market for cryptocurrencies by providing a comprehensive analysis of short-term (over)reactions that follow significant price changes of such currencies.

We identify and analyze overreactions and mispricing in markets for crypto currencies by applying a broad set of thresholds that depend on market specific dynamics and volatilities. We analyze the returns on days following abnormal returns and identify significant differences from normal returns using the t-test and the Mann-Whitney-U-test. We further complement the literature by using endof-the-day returns in addition to high-low returns. Additionally, we consider a broad sample of 50 cryptocurrencies for an expanded time span (2015 to 2020) that includes the big currencies as well as smaller currencies.

We detect the existence of overreactions and, thus, market inefficiencies in crypto markets. The findings for different methodological approaches are similar, which underpins the robustness of the findings. By considering a broad sample that includes small and big currencies we can show the existence of a market size effect. By considering a broad set of thresholds, we further found evidence for a magnitude effect, which means that higher initial abnormal returns are related to higher inefficiencies.

Our paper has practical implications. We detect market inefficiencies that can be used in practical trading to obtain excess returns. In fact, our methodological approach and its results can be used to derive a strategy for trading in cryptocurrencies that can be easily implemented. Based on our findings, we can expect positive access returns by applying this trading strategy.

We complement the literature on market inefficiencies and mispricing in crypto markets by analyzing price patterns after initial abnormal returns. We contribute by applying different methodological approaches in addition to the approaches used so far, by considering a set of different thresholds, and by applying a much broader data set that enables us to analyze additional aspects.
\end{abstract}

\title{
1 Introduction
}

The market for cryptocurrencies is a newly created market that differs fundamentally from other capital markets. There is no central bank or other state institution that issues and backs a cryptocurrency. In many countries, these currencies are very weak or unregulated. Institutional investors, such as banks or mutual funds, have mostly avoided investments in cryptocurrencies due to the high level of volatility and the resulting risks. In contrast, the cryptomarket is very attractive for some investors, especially private investors, because of the high return-risk profile and perhaps also because of the low government requirements and regulations. Compared to other investment opportunities, e.g., stocks, bonds or commodities, cryptocurrencies are not associated with any material or ideational values. In contrast to a stock, the price of a cryptocurrency does not reflect material or immaterial values, such as factories, buildings or knowledge and patents. Since cryptocurrencies have no intrinsic economic value, they cannot be assessed by any fundamental values. The only economic factors for the price are the confidence of investors and the shortage of coins. These facts can have very different implications with regard to market efficiency. On the one hand, a reversal to the fundamental value after an overreaction is scarcely possible, since this fundamental value does not exist or is hardly possible to derive. On the other hand, the absence of fundamental values can increase irrational price movements, such as a high level of volatility and overreactions. These differences from ordinary capital markets make an investigation of the efficiency of cryptomarkets interesting. One might suspect that 
cryptomarkets are less informationally efficient than markets for other asset classes because of the mentioned features. Our paper contributes to the literature on the efficiency of cryptomarkets. We particularly consider the price reaction following abnormal events, especially large price changes. We look for the existence of systematic patterns after days with abnormal price changes in the cryptomarket. Empirical evidence of significant patterns would be an indication against the efficient market hypothesis. If such a pattern were observed, then the investors could exploit it. This, however, would destroy such a pattern and make the potential investment strategies unprofitable. Our paper analyzes the market reactions following abnormal returns. While comprehensive literature on market efficiency exists for other asset classes that includes a variety of papers on price reactions after abnormal returns, this specific but important issue has rarely been analyzed in cryptomarkets. Only a few papers exist, which we discuss together with our contributions in the next section.

We contribute to the literature in several ways. First, we consider a broad set of thirty cryptocurrencies by employing time series that are as long as possible, whereas most of the scant literature on cryptocurrencies focuses on a few currencies, generally the largest. Thus, we can also analyze the issue of market efficiency for small cryptocurrencies for which the markets are supposed to be narrower. In doing so, we realize our second contribution of analyzing the effect of market size on the efficiency of cryptomarkets. In addition, we analyze whether a magnitude effect exists, i.e., whether higher initial abnormal returns lead to higher market inefficiencies in the following days. Finally, we contribute by using different methodological approaches in addition to those already used in the literature. Although our research is based on the inspiring existing methodology, we use a somewhat modified approach, which makes our results more realistic with respect to exploitable trading strategies.

In fact, our methodological approach and its results can be used to derive a strategy for trading in cryptocurrencies that can be easily implemented. Based on our findings, we can expect positive access returns by applying this trading strategy.

The state of the existing literature is described in more detail in the following section. Based on this information, section 3 describes our contribution and the methodological approach applied in this study in detail. In section 4, the empirical results are presented. Finally, section 5 concludes all findings.

\section{The literature and our contribution}

\subsection{Price overreactions on the capital market}

Overreactions have been observed and confirmed in many different markets and countries. A first paper from De Bondt and Thaler (1985) investigated long-term overreactions in the New York stock market. They found that stocks with a below-average return produced significantly higher returns in the following period. This effect is known in the literature as the winner-loser effect. Brown et al. (1988) found that the stock market overreacted after bad news based on the 200 largest stocks in the S\&P 500. However, Brown et al. viewed these anomalies as confirming the efficiency market hypothesis, since the abnormal returns merely reflected the increased risk after an event. The rational investor demands a higher risk 
premium as a kind of compensation for the increased risk or volatility (risk change hypothesis).

An early study on short-term overreactions, which was published by Arbel and Jaggi (1982), could not find any significant patterns after extreme price movements. They investigated the return behavior of 180 stocks in the 11 days before and 10 days after the stock's appearance on the Wall Street Journal's daily price leaders' list. Further studies on overreactions in the US stock market are reported by Atkins and Dyl (1990), who examined overreactions in the New York Stock Exchange for the period 1975-1984 and detected that price overreactions in the negative direction have a higher reversal than in the positive direction, but both reactions are hardly exploitable; by Cox and Peterson (1994), who confirmed overreactions for the period 1963-1991 and explained the detected size effect as a possible proxy for the bid-ask bounce; by Larson and Madura (2003), who showed in the period 1988-1995 that stocks tend to overreact after uninformed events but not after informed events; and by Ma et al. (2005), who found strong evidence for price reversals after overreactions in the price history of Nasdaq stocks between 1996 and 1997. Caporale and Plastun (2019) examined not only the US stock market in terms of short-term overreactions but also the Foreign Exchange (FOREX) and commodity markets. They confirmed overreactions in the stock market as well as partly in the FOREX and commodity markets. However, a trading simulation showed that an exploitation of these overreactions is not profitable. Delayed reactions or underreactions were statistically more significant in their results. By exploiting these effects (which are called "inertia anomalies" by the authors), the trading simulation generated higher profits. Over time, an increasing number of scientific studies have found anomalies in the capital market that could not be explained by the efficient market hypothesis. Therefore, several economists have tried to explain these effects with other theories, such as behavioral finance. Thus, Daniel et al. (1998) proposed a theory of market under- and overreactions, which is based on two psychological biases: overconfidence and biased self-attribution. In their theory, they "define an overconfident investor as one who overestimates the precision of his private information signal, but not of information signals publicly received by all." This overestimation of private signals of investors can lead to mispricing of assets and consequently to deviations from the fundamental value. Furthermore, the authors suggest that self-attribution bias may extend overreactions over time. This bias describes the tendency that investors attribute successes to their own abilities and losses to external circumstances. Due to this bias, "new public signals on average are viewed as confirming the validity of his private signal. This suggests that public information can trigger further overreaction to a preceding private signal." Nonetheless, a reversal effect is observable in the subsequent time: "on subsequent dates, as more public information arrives, the price, on average, moves still closer to the full-information value." Hence, the self-attribution bias can cause "short-run momentum and long-term reversals."

Short-term overreactions have been investigated in many other countries. For example, Lobe and Rieks (2011) detected overreactions in the German stock market. Their results showed that overreactions occurred, but these were hardly exploited due to transaction costs. In addition, there were signs of size effects in their findings, since overreactions occurred more frequently in the price movements of stocks, which are listed in smaller indices. Spyrou et al. (2007) looked for overreactions in the UK stock market. Their results indicated that in large capitalization indices, the markets react efficiently. They did not measure significant abnormal returns after extreme events. Nevertheless, they also discovered that stocks listed 
in medium and small indices tend to underreact. The authors explain these underreactions with the argument that "size is a proxy for factors such as availability of information to holders of medium and small capitalization equity securities and/or a reduced number of analysts covering these stocks." An investigation of the Japanese stock market was performed by Chang et al. (1995). With the help of portfolio constructions for the period 1975-1991, they examined the short-term profitability of contrarian investment strategies by purchasing in falling markets and selling at high market prices. They confirmed a significant profitability of the contrarian strategies but stated that neither the size of the firms nor the return seasonality can fully explain the short-term contrarian profits.

\subsection{The efficiency and overreactions on the cryptomarket}

In recent years, not only has the interest of investors in cryptocurrencies increased but scientific interest has also increased. In addition, some articles have appeared that address efficiency in the cryptocurrency market. The first article devoted to the efficiency measurement of Bitcoin comes from Urquhart (2016). With several statistical tests, he examined the returns of this cryptocurrency in terms of autocorrelation, independence and randomness (random walk). His research confirms the strong inefficiency of Bitcoin. However, Nadarajah and Chu (2017) reviewed Urquhart's results with transformed Bitcoin returns. They found that the Bitcoin price can be considered weakly efficient with this method. Bariviera et al. (2017) found an improvement in the efficiency of the Bitcoin price over several years. The results of the efficiency measurement from Latif et al. (2017) were also inconsistent with the weak form of the efficiency market hypothesis.

Furthermore, several articles have addressed the day-of-the-week effect in connection with cryptocurrencies. Décourt et al. (2017) showed that Bitcoin returns on Monday are higher than on other days. Nevertheless, they concluded that it is almost impossible to exploit this effect with any trading strategy. Likewise, Kurihara and Fukushima (2017) found anomalous daily effects and thus refuted market efficiency. However, their results confirmed that the efficiency improved over time. Caporale and Plastun (2018) also confirmed the Monday effect on Bitcoin returns. They showed with the help of a trading simulation that an exploitation of this effect would have been profitable. On the other hand, they examined three other cryptocurrencies, but they could not confirm such an effect in the price history of these coins. These findings are consistent with the study by Aharon and Qadan (2018), who confirmed the existence of the day-of-the-week anomaly not only in returns but also in the volatility of Bitcoin by using ordinary least squares (OLS) and generalized autoregressive conditional heteroskedasticity (GARCH) models.

Effects related to behavioral economics have also been considered in empirical studies. For example, Poyser (2018) investigated herd effects. His findings suggest that investors deviate from rational pricing, which is contrary to market efficiency. Using a rolling-window analysis, Bouri et al. (2018) also confirmed herding behavior in the cryptomarket. In addition, they found that herding behavior occurs with increasing uncertainty. Similarly, Murray Leclair (2018) found empirical evidence for herding behavior in his investigation of 12 cryptocurrencies.

To date, only a few articles have been published that investigate overreactions or abnormal short-term price patterns in the cryptomarket. Caporale and Plastun (2019) studied patterns in the price history of four cryptocurrencies with the help of various statistical tests ( $t$ test, ANOVA, regression, Mann-Whitney U test) in the period 2013-2017. The empirical results 
confirmed their hypothesis that the counterreactions after one day with a very high volatility differ significantly from the price changes after normal days. However, an investigation with a trading strategy revealed that the evidence found on overreactions would not have been exploitable. The methodological approach applied by Caporale and Plastun is a proper and expedient basis for research on short-term overreactions. Thus, we apply this approach and a modified version in our analysis. However, other approaches to identify short-term overreactions in the cryptomarket are also used in the literature, which we briefly consider in the following.

In another article, Caporale and Plastun (2020) analyzed the price history of the three coins with the highest market capitalization by applying a cumulative abnormal returns (CAR) approach. They considered momentum and contrarian effects in hourly returns on the overreaction and the postoverreaction day. For this purpose, they also used a $t$ test and a trading simulation. Their results mostly suggest the existence of a momentum effect on event and postevent days with regard to hourly returns. In two cases, they found indications of a contrarian effect in hourly price behavior. Chevapatrakul and Mascia (2018) used quantile autoregressive models. In contrast to Caporale and Plastun (2019) and other papers mentioned in the following, they considered Bitcoin only in their analysis covering the time between 2013 and 2018. They found evidence of overreactions. According to their findings, overreactions appeared during days of sharp declines in the Bitcoin price and during weeks of market rallies.

Borgards and Czudaj (2020) chose a different approach to detect interday and intraday overreactions for 12 cryptocurrencies. A moving-average smoothing filter algorithm identifies overreactions, which are defined as price changes from a turning point to its subsequent turning point, for six different frequencies ( 1 day to 30 minutes). In the next step, the Mann-Whitney U test compares the overreaction with the subsequent reversal. The advantage of this method is that the definition of an overreaction is not bound to a time limit, which allows the investigation of the whole extent of the overreaction. The drawback of this approach is that the results are dependent on the smoothing and frequency parameters, which makes a general interpretation of the results difficult. However, for the most part, their empirical findings are in line with those of the papers mentioned above and strongly support the overreaction hypothesis. The applied trading strategy is even profitable after transaction costs, which is a violation of the efficient market hypothesis. Corbet and Katsiampa (2018) also observed mean-reverting patterns in the Bitcoin price history by estimating ANAR and EGARCH models. Kosc et al. (2019) reviewed momentum and contrarian effects in the cryptomarket using portfolio compilations and included the 100 coins with the largest market capitalization. Their results show that the contrarian effect exceeds the momentum effect and the benchmark portfolios. Karalevicius et al. (2017) examined the influence of cryptorelevant articles on the Bitcoin price by measuring the number of positive and negative words in the article. Their findings suggest an interaction between media sentiment and the Bitcoin price. They also found that investors overreact to news given at short notice.

\section{Data and Methodology}

The applied methodology in our paper is inspired by the approach that is used by Caporale and Plastun (2019). Motivated by this inspiring paper, we contribute to the literature in 
several ways. First, we made some important changes in the methodological approach. In particular, we use a modified approach to calculate daily returns. In addition, we consider the market reaction on several days following an initial abnormal return. We further contribute by analyzing the market reaction after events that comprise more than one day with abnormal returns, i.e., a row of two or three days of abnormal returns. Our methodological approach and its application are explained below in detail. However, since both approaches have their pros and cons, we apply our modified approach with the approach used by Caporale and Plastun (2019), which enables us to compare the results and provide a more comprehensive picture of the issue.

Second, in addition to the methodological changes, we contribute by considering a far broader set of cryptocurrencies and a longer time span. While Caporale and Plastun (2019) consider four currencies, our sample comprises fifty currencies. These are the cryptocurrencies with the highest market capitalization on 07-01-2020. For our investigation, we use daily price data in the period from 01-01-2015 to 12-31-2020, downloaded from coinmarketcap.com. Since many cryptocurrencies are relatively new, the data availability of some of these coins starts later. The investigation period of each coin can be found in Table 1 in the appendix. The broad data sample enables our third contribution, namely, the analysis of the impact of market size on the results. We also include minor coins with less market capitalization and trading volume. We are able to analyze whether market inefficiencies exist in smaller markets and whether there are differences with respect to market size.

In the following section, we discuss our contribution regarding the methodological approach in detail. As already mentioned, we contribute to the literature by applying changes in the research approach that have led to some advantages. First, it enables us to distinguish between positive and negative overreactions, whereas Caporale and Plastun (2019) do not distinguish between both cases and include them together in the empirical analysis. Thus, our approach leads to a more precise analysis and more informative results. Moreover, our approach can be considered to be more realistic with respect to potential trading strategies and the potential exploitation of market inefficiencies.

To explain this, we first describe the methodology in more detail. Thus, we return to the contribution of our paper later. The goal of this article is to detect systematic patterns on the days following abnormal daily price changes in the cryptomarket. The theoretical assumption is that abnormal price changes can be viewed as overreactions or underreactions. It is therefore assumed that the price returns to its fundamental value on the following day (mean reversion) or the price is further increased on the following day (momentum). If there are significant price movements in the reversed direction on Day $t+1$ after an abnormal price change on Day $t$, the price change on Day t can indicate overreactions. Conversely, if there are significant price movements in the same direction on Day $t+1$, the price change on Day t can indicate underreactions. If no overreactions or underreactions occur, no significant patterns will be measurable on the following day. The latter assumption will represent the null hypothesis:

H0: The price change on the day after an abnormal daily price change does not differ significantly from the price changes on days that follow days with normal price changes. 
From the previous overreaction (or underreaction) assumption, the following alternative hypothesis can now be derived:

H1: The return (or rather the counterreaction "cR") on the day after an abnormal price change is significantly different from those price changes after normal days.

The first step is the calculation of the returns for all cryptocurrencies. The returns are defined as the difference between the closing price on Day $t$ and the previous closing price on Day t1 in percent. They are calculated as follows:

$$
R_{t}=\ln \left(\frac{\text { close }_{t}}{\operatorname{clos}_{t-1}}\right) \times 100
$$

where $\mathrm{R}_{t}$ is the return in percent, close $\mathrm{t}_{t}$ is the closing price on Day t and close $\mathrm{t}_{t-1}$ is the closing price on Day t-1. This calculation is applied for all observation days, regardless of whether we consider event days or postevent days. The calculation of the returns is one major difference and contribution of our paper. We use closing prices, whereas Caporale and Plastun (2019) calculate the daily returns as the difference between highest and lowest prices (which can be detected ex post but not during the trading day). In the original approach of Caporale and Plastun, the returns are the difference between the high and low prices:

$$
R_{t}=\ln \left(\frac{\text { high }_{t}}{\operatorname{low}_{t}}\right) \times 100
$$

However, only the results after positive abnormal price changes can be calculated with this method since the difference between the daily high and the daily low of the price is always in the positive range. Nevertheless, we additionally apply the approach of Caporale and Plastun. This enables us to compare the results and provide robust and comprehensive results for different measurement approaches. The counterreactions or postevent returns are calculated using the approach of Caporale and Plastun as follows:

$$
R_{t}=\ln \left(\frac{\text { open }_{t}}{\text { low }_{t}}\right) \times 100
$$

A second difference from the mentioned article is the use of thresholds to identify abnormal returns. Similar to Caporale and Plastun (2019), we consider no absolute thresholds but thresholds related to the standard deviation, i.e., the volatility of the respective time series of returns. They considered the threshold of only one standard deviation. We also use this threshold but apply a broader approach to provide a wider view of the issue. To detect the magnitude of overreactions in our study, eleven thresholds from 1 to 6 in 0.5 steps $(\mathrm{k}=1,1.5$, $2,2.5,3,3.5,4,4.5,5,5.5,6)$ are initially defined. These thresholds are multiplied by the standard deviation of the returns of the entire period. The 11 thresholds were chosen because they provide a good overview of the magnitude of abnormal price shocks. A threshold for a daily price change should be at least one standard deviation to be considered abnormal. We do not consider higher thresholds, although we could suspect more pronounced price patterns following them, since for higher thresholds, there are too few observations for a statistical test. The calculation of abnormal price changes does not use static values, such as 5 or 10 percent, but dynamic thresholds, which are expressed as 
standard deviations. Since each coin has a different volatility, this method has the advantage that the threshold adjusts to the respective volatility. A positive abnormal price change "aR" (or abnormal return) on event Day t is described as follows:

$$
a R_{t}>(\bar{R}+k \times s)
$$

A negative abnormal price change in the opposite way:

$$
a R_{t}<(\bar{R}-k \times s)
$$

where $\mathrm{k}$ is the number of standard deviations (thresholds) and $\bar{R}$ is the average of all returns $R_{t}$ for the entire study period $\mathrm{n}$ :

$$
\overline{R_{t}}=\sum_{t=1}^{n} \frac{R_{t}}{n}
$$

$s$ is the standard deviation of the returns of the whole sample:

$$
s=\sqrt{\frac{1}{n} \sum_{t=1}^{n}\left(R_{t}-\bar{R}\right)^{2}}
$$

After the calculation has been performed for each cryptocurrency, the observations of all coins are combined into a pooled dataset. This means that the evaluation is done for the top of the whole cryptomarket (top 50 cryptocurrencies) and not for individual coins. The advantage here is to obtain more general and statistically more meaningful results. To detect the existence of patterns after abnormal price changes, two statistical tests are performed: Welch's t test, which is used for unequal variances and unequal sample sizes:

$$
t=\frac{\overline{c R}-\overline{n R}}{\sqrt{\frac{s_{c R}^{2}}{N_{c R}}+\frac{s_{n R}^{2}}{N_{n R}}}}
$$

and the Wilcoxon-Mann-Whitney U test, which compares the rank sums of the postevent returns and the returns after normal price changes. The advantage of this test is that it can also be used for nonparametric distributions. These tests compare the returns after days with an abnormal price change, i.e., the potential "counterreactions," that we, thus, call "cR," with the returns after days with normal price changes " $\mathrm{nR}$ " (the nR-days thus describe all days except the cR-days). The return or counterreaction $c R_{t+1}$ on the postevent Day $\mathrm{t}+1$ is also given as the percentage change of the closing price and the previous closing price:

$$
c R_{t+1}=\ln \left(\frac{\operatorname{clos}_{t+1}}{\operatorname{close}_{t}}\right) \times 100
$$

Since we have explained our methodological approach, we can discuss in more detail than above how it differs from the approaches applied in the literature, especially Caporale and Plastun (2019), together with the advantages and disadvantages of both approaches. For the 
calculation of the $\mathrm{cR}$ returns, Caporale and Plastun use the difference between the opening price and the low price after increased price changes and the high and open price after decreased price changes, whereas we use the difference between the closing price and the previous closing price. Naturally, their type of calculation leads to higher values and more significant results since it considers the largest possible difference in prices. This is suspected to provide results that are more significant. This approach could be based on the assumption of perfectly informed investors, who are able to identify high and low prices. If one wants to be sure that the rejection of market inefficiencies is valid, this approach is the first choice. However, it has some drawbacks. First, this calculation method can be considered somewhat unrealistic with respect to practical trading. It is virtually impossible for a real-world investor to determine ex ante the moment for the high as well as the low price, and thus, it is not possible to apply a trading strategy based on these values in practice. Thus, by using the approach, one may overestimate exploitable market inefficiencies. Our approach, by contrast, is rather conservative, since it is based on a simple trading strategy-buy at the closing price on Day t and sell at the closing price on the next Day $\mathrm{t}+1$ - which leads to more robust results; i.e., if we detect significant inefficiencies, they can be profitably used with a simple strategy. Moreover, our approach enables us to distinguish between positive and negative abnormal price changes. In Caporale and Plastun (2019), by contrast, only positive abnormal returns can be considered. Since their calculated returns $\left(R_{t}\right)$ are the difference between the high and the low price, consequently, all returns are in the positive range. Hence, it is possible to examine only abnormal price reactions in the positive direction. Our paper uses the difference between the open and close to distinguish between abnormal price changes in both the positive and negative directions.

As explained above, our paper also uses different thresholds to identify abnormal returns, while Caporale and Plastun (2019) consider only one threshold, a price change higher than one standard deviation. Thus, our approach provides a more comprehensive view of the issue. Moreover, the help of different thresholds facilitates determining a magnitude effect, i.e., whether a higher initial abnormal price change leads to higher reactions on the following day. From this assumption, the following hypothesis can be derived:

H2: The higher the abnormal price change $a R_{t}$ on Day $\mathrm{t}$ is, the higher the return $c R_{t+1}$ on the following Day $t+1$.

Another contribution concerns the period for which overreactions are checked. We extend the existing literature by considering not only the price reaction on Day $t+1$ but also a possible delayed reaction on the five following Days $t+1$ until $t+5$. Thus, we address the possibility that price reactions do not occur on Day $t+1$ but on the following days. We consider two types of results. On the one hand, we use the results of the individual postevent returns $t+1 \ldots t+5$. On the other hand, we look not only at individual returns for single days but also at returns over periods of multiple days. Hence, we consider the sum of the following postevent returns $\mathrm{t}+2$ to $\mathrm{t}+\mathrm{X}$ (with $\mathrm{X}=2,3,4,5$ ).

We contribute further by investigating whether the price reaction $\mathrm{cR}$ after abnormal price changes is more significant or higher if two or three consecutive abnormal price changes occur in the same direction. To the best of our knowledge, an analysis that considers reactions of cryptomarkets after several days with consecutive abnormal returns has thus far not been done in the literature. If there is an abnormal price change $a R_{t}$ on Day $t$ and on the following Day $t+1$ (and $t+2$, respectively), an abnormal price change also occurs that is higher 
than one standard deviation; then, the assumption is that the counterreaction cR on Day $\mathrm{t}+2$ (or $t+3$, respectively) is significantly different from the price changes after normal days. These assumptions are summarized in Hypothesis 3:

H3: If abnormal price changes occur on two or three consecutive days in the same direction, then the price change on the following day ( $t+2$ or $t+3$, respectively) is significantly different from that after normal days.

Finally, we contribute by examining the magnitude effect. We do so by using OLS regressions. The return on postevent Day $t+1$ represents the dependent variable. The independent variables are the abnormal return on Day $t$, the trading volume on Day $t$ and the market capitalization of the coin on Day t. Thus, we are also able to investigate whether trading volume and market capitalization affect the postevent returns.

\section{Empirical Results}

The investigated coins are shown in Table 1. This table provides further information such as the price, the market capitalization and the start of the investigation period. Furthermore, descriptive statistics such as the mean and the standard deviation of the daily returns of all coins are included. This shows that the return volatilities of the coins are different. The coin Filecoin has the highest volatility of the daily returns with a standard deviation of 10.91 . In contrast, the volatility of USD Coin is the lowest, with a value of 0.51 . This substantial difference in the volatility of the considered time series underpins that it is important to use volatility-related thresholds that are better suited to the problem than fixed percentage numbers.

\subsection{Results obtained using end-of-the-day returns}

\section{Test of abnormal returns on postevent-Day $t+1$}

We start with the results after abnormal price changes in the positive direction. Table 2 in the appendix summarizes these results. The table shows that the majority (7 of 11 thresholds) of the average returns on the postevent day are in the positive range. This means that after a positive abnormal price change, the price on the following day also increases in most cases. However, we observe mixed results and a U-shaped pattern. From a threshold of 4.5 upward, the $\mathrm{cR}$ values are negative; however, no cR return is significant according to the $t$ test, while according to the Wilcoxon test, nine values show statistical significance. Therefore, the evidence with respect to Hypothesis $\mathrm{H} 1$ is mixed. The positive sign of the mean counterreaction indicates that the initial positive abnormal price change is an underreaction to the information, since further positive changes follow on the next day. It is interesting to see that up to the threshold of 3.5, an ascending pattern can be observed. The mean cR values increase with rising thresholds. Thus, we observe a magnitude effect for these thresholds, which is evidence in favor of Hypothesis H2. However, at even higher thresholds (from threshold 4 onward), this pattern reverses into the negative range. In contrast to the results for lower thresholds, these values for higher thresholds are no longer statistically significant. The picture is different for abnormal price changes in the negative direction, illustrated in Table 3. All mean cR values are highly significant according to the test and the Wilcoxon test. 
Thus, Hypothesis $\mathrm{H} 1$ is strongly supported for negative abnormal returns, i.e. also in the case of (negative) abnormal price changes, the returns on the following day (cR values) are significantly different from those price changes after normal days. Moreover, since all mean cR values are significant and show a positive sign, we find strong evidence of counterreactions following abnormal negative price changes. This indicates an overshooting of the markets on the information that leads to the initial abnormal negative return. Furthermore, most mean cR values increase with higher thresholds. This fact supports Hypothesis H2. Thus, the existence of a magnitude effect is also confirmed for negative abnormal price changes. In this case, the results for all thresholds are significant, and we observe this pattern for the entire span of thresholds.

\section{Test of abnormal returns on later days}

Figure 1 displays the results after abnormal price changes in the positive direction for the 5 following postevent days graphically. The results for Day $t+1$ after both positive and negative abnormal price changes have already been displayed in Table 2 and Table 3. With the help of this examination, it can be shown whether delayed price reactions occur on Day $t+1$ until $t+5$. To measure the significance Welch's $t$ test and the Wilcoxon-Mann-Whitney $U$ test, are performed as described above. The results are mixed. The returns on Days $t+2$ to $t+5$ are usually not as high as those on Day $t+1$. However, we observe some significant mean $c R$ values. According to the t test (the significant values are marked with filled circles), 10 out of 44 tests are significant. The Wilcoxon-Mann-Whitney $U$ test led to 7 significant results. In general, tests for lower thresholds, for which we have more observations, are significant.

Similarly, the results for negative abnormal returns are mixed. However, we observe more significant cases. In Figure 2, 15 out of 44 mean $c R$ values of the postevent Days $t+2$ to $t+5$ are significant after abnormal price changes in the negative direction. For our nonparametric test, the number of significant cases is much higher, and 28 of 44 cases are significant. Thus, we observe a counterreaction to the counterreaction. As discussed above, we observe a positive counterreaction on the initial negative abnormal return. This positive counterreaction is highly significant but restricted on the first postevent day. On the following days, we observe a negative return, i.e., a counterreaction to the counterreaction. The postevent returns on Days $t+2$ to $t+5$ are likewise lower than those on the first day. The results with respect to significance are mixed. A magnitude effect seems to affect not only Day $\mathrm{t}+1$ but also Days $\mathrm{t}+2$ and $\mathrm{t}+4$.

Does the picture change if we consider the postevent returns calculated over periods that comprise several days? By considering average returns calculated from several days following the event, we acknowledge that a delayed counterreaction can occur not only on one day but also on two or more days. This means that the reaction is slower and distributed over more days. Moreover, our results from above may be hampered by the fact that in some cases, the delayed reaction follows on the second day, while in other cases, it follows on the third day or even on later days. To investigate this assumption, we additionally consider postevent returns over several postevent days. For the returns after normal days (nR), we calculate the value of $n R$ for the respective period in the same way that the $\mathrm{cR}$ returns were computed previously. Subsequently, the $\mathrm{cR}$ and $\mathrm{nR}$ values calculated for these extended periods are analyzed with both statistical tests in the same way as for single days. The results and their significance are shown in Figure 3 and Figure 4. To avoid distortions, the postevent 
Day $t+1$ was omitted. For example, the $c R$ value on postevent Day $t+4$ represents the sum over the daily returns on Days $t+2, t+3$ and $t+4$.

Figure 3 shows that after positive abnormal price changes, approximately half of the $c R$ returns (23 of 44 values according to the test, 15 according to the Wilcoxon test) are significant. Therefore, again, the results are mixed. There is some but no overwhelming evidence for delayed reactions. In Figure 4, where the average returns after negative abnormal price changes are shown, we observe even more significant cases (35 of 44 values according to the $t$ test, 41 according to the Wilcoxon test). Some values on Days $t+4$ and $t+5$ are relatively high. In addition, all significant average returns are in the negative range, especially at higher thresholds. As shown in Figure 2, after a negative abnormal price change, there is a very strong and positive counteraction on Day $t+1$. Thus, the negative average returns on Days $t+4$ and $t+5$ in Figure 4 seem to be a counteraction on the counterreaction, i.e., to the significantly positive counteraction on postevent Day $t+1$. This may indicate that after a negative overreaction on Day $t+0$, the subsequent positive counteraction on Day $t+1$ is also an overreaction.

\section{Abnormal returns following days with consecutive abnormal initial returns}

Now, we discuss the results after two and three consecutive days with abnormal price changes in the same direction. Because only a few events with two and three consecutive days were observable, the threshold with one standard deviation was applied to obtain enough observations. Table 4 shows that we obtain results similar to those obtained thus far: All mean cR values are in the positive range regardless of whether we analyze positive or negative initial abnormal price changes. The average $\mathrm{cR}$ returns are higher in most cases compared to those observed after initial abnormal returns for single days with threshold $\mathrm{k}=$ 1 (see Table 2) and Table 3). However, there is some lack of significance, at least for the case of three consecutive abnormal returns, which may be due to the low number of observations. In the case of 2 positive consecutive abnormal price changes, only the Wilcoxon test shows a significant difference. The mean return after 2 consecutive days with a negative abnormal price reaction is highly significant according to both tests. With an average return of 2.62, this value is relatively high. For three consecutive abnormal returns, the results are not significant according to the t test. Consequently, with respect to Hypothesis H3, the results are mixed.

\section{Explaining postevent returns with regression models}

The next section of the results uses an OLS regression to examine which factors can explain the postevent returns. We consider both cases, i.e., positive and negative abnormal price changes in separate regressions. We use the lowest threshold to identify aR returns and include all $\mathrm{aR}$ and $\mathrm{cR}$ values starting from one standard deviation $(\mathrm{k}=1)$ in the regressions. We apply the three explanatory variables in a regression in which the postevent return is the dependent variable. The initial abnormal return that defines the event is our first explanatory variable. Thus, we test whether a magnitude effect exists, i.e., whether higher initial abnormal returns lead to significantly higher postevent returns. In addition, we test the size effect, i.e., whether "smaller" coins lead to higher market inefficiencies, i.e., lower (in absolute terms) postevent returns. Here, we consider two variables, trading volume and market 
capitalization. The former accounts for the actual market size and market liquidity by considering the coins that are actually traded, while the latter measures the market potential since it is related to the volume of coins that could be traded.

To run proper regressions, some technical issues must be considered and solved. Both the trading volume and the market capitalization are closely related to the price of the coins, since they are calculated as the market price multiplied by the number of coins traded (trading volume) and the market price multiplied by the number of coins in circulation (market capitalization). Since the (change in the) price determines the return, there is an endogeneity issue if the return from the same day is the left-hand side variable. Thus, we consider instead of the current trading volume or market capitalization (which depends on the current price change) on Day $t+1$ the (moving) averages for trading volume and market capitalization for the 20 days before, i.e., Day $\mathrm{t}-19$ to Day $\mathrm{t}+0$. This provides a measurement of the general level of trading volume and market capitalization in the respective market, which is independent of the price change on the current date. Both quantities are closely related, yet they are used as measures for the same issue, namely, the size of the market. Thus, multicollinearity is an issue. To address multicollinearity, we run univariate regressions in which the variables are considered separately. Due to existing heteroskedasticity in both models, which is determined by the Breusch-Pagan test, the regressions are performed with robust standard errors (HC3).

Table 5 shows the results for the case of positive abnormal returns. As indicated in Table 2 , the abnormal return "aR" has no significant effect on the postevent return "cR," which therefore does not confirm the existence of a magnitude effect in the positive case. This, in some sense, confirms the results shown above, where we admittedly observed a magnitude effect for smaller thresholds but overall a U-shaped dependency and insignificant results for higher thresholds. The (moving average of the) trading volume is highly significant and has a negative sign. This means that the lower the trading volume in the respective market is, the higher the market imperfection, which results since the initial market reaction on the causative information is not large enough (underreaction). This market size effect is confirmed by the significant impact of the (moving average of) market capitalization. The negative sign indicates that the smaller the market capitalization of the coin is, the higher the postevent return and the higher the market imperfection.

For the case of negative abnormal returns, we obtain different results (see table 6). The trading volume still has a negative sign, but the impact is not significant in this case. In contrast, both of the other variables, the initial abnormal return and the market capitalization, are significant. For the discussion of the impact of the initial abnormal return "aR" on the observed return on the following day (which is the dependent variable), it is important to keep in mind that the initial abnormal return is negative in this case. Since we observe a highly significant negative sign of the abnormal return "aR" in the regression, it can be concluded that the lower the abnormal return on Day $t$ is, i.e., the higher in absolute terms, the higher (positive sign) is the postevent return "cR" on the following day. This means that the counterreaction following a negative abnormal return is larger, and thus, the market imperfection is higher. This is a strong indication of the magnitude effect and a violation of the efficient market hypothesis. The (moving average of the) market capitalization has a significantly negative sign. This confirms the existence of the "size effect" because coins with a larger market capitalization have lower postevent returns; i.e., for larger coins, the 
counterreaction (price increase) after initial negative abnormal returns is lower, while for smaller coins, it is higher.

\subsection{Results obtained using alternative calculation of returns by considering high and low prices}

The second part of this section is dedicated to the results with the calculation method of the returns provided by Caporale and Plastun (2019), where instead of the end-of-the-day prices, the high and the low prices are used.

\section{Test of abnormal returns on postevent-Day $t+1$}

Table 7 shows the results for positive abnormal price changes, similar to Table 2 above. As explained, initial negative abnormal returns are not considered. As expected (as explained above), the changed calculation method leads to a higher level of all returns for both the normal returns (nR) and, in particular, the cR returns after abnormal price changes. The difference between the postevent returns (cR) and the returns after normal price changes $(\mathrm{nR})$ is likewise much more significant. This applies to all thresholds. Only at threshold 1 is the postevent return not significant according to the Wilcoxon test. These results clearly indicate the existence of market inefficiencies.

The postevent returns constantly rise with increasing thresholds. With threshold 6, the postevent return is 16.5 percent, which is nearly two times higher than the return after normal days (8.2 percent). A magnitude effect is therefore clearly visible in the case of the considered high-low returns, which is less obvious for end-of-the-day returns considered above. For end-of-the-day returns, we find evidence for a magnitude effect for the case of negative initial abnormal returns, whereas the results are rather mixed for positive initial abnormal returns.

\section{Test of abnormal returns on later days}

Additionally, for days later than $t+1$ after the event, the returns are higher, and most of the results are significant. Figure 5 shows the results for positive abnormal returns. The largest price changes occur on postevent Day $t+1$. However, on later days, we also observe considerable returns, which decrease slightly on the following days. Overall, 30 of 44 values in the postevent period $t+2$ to $t+5$ are significant according to the $t$ test (and 30 values are significant according to the Wilcoxon test). On postevent Day $t+5$, only one value is still significant. Additionally, a magnitude effect is visible on Days $t+2$ to $t+4$; i.e., for higher thresholds, the effects are higher.

\section{Abnormal returns following days with consecutive abnormal initial returns}

Table 8 summarizes the findings after 2 and 3 consecutive days with a positive abnormal price change. As in Table 4, only threshold 1 is applied here. This table shows that all postevent returns on Days $t+2$ and $t+3$ are significant except for the $t$ test at consecutive Day $t+2$. In addition, the returns are slightly higher than those reported (for threshold 1) for the

case of initial abnormal returns that occur on a single day, which we considered thus far (see 
Table 7). This indicates that the more abnormal price changes follow each other, the more inefficient the market will react on the next day.

\section{Explaining postevent returns with regression models}

Finally, the univariate OLS regressions in Table 9 confirm the existence of a magnitude effect due to the significant estimate of the "aR" value $(\mathrm{p}<0.05)$. Moreover, we confirm the market size effect for the case of high-low returns, which was already detected for end-of-the-day returns, since the volume again has a significantly negative effect. Market capitalization also seems to have a significant influence on high-low returns.

\subsection{Discussion of results and their practical implications}

Our results have several important implications for both practical trading and scientific research on market imperfections. The existence of significant excess returns shows that cryptomarkets are not informationally efficient. The significant market size effect shows that markets for smaller currencies are less efficient than markets for larger currencies.

These inefficiencies and the observed excess returns imply that trading strategies can be developed for which positive excess returns can be expected. The advantage of our methodological approach compared to alternative approaches in calculating (excess) returns (see the discussion in Section 3) is that it can be directly transferred in a simple trading strategy. The simple trading strategy that directly corresponds to our results would follow the simple rule: "By on the end of a trading day were excess returns are observed and sell on the end of the following day". It does not matter whether the initial excess return is positive or negative, since in both cases, a significant positive return is expected on the following day. Our results indicate that we can expect significant positive returns by applying this trading strategy. The significant market size effect indicates that higher success of this strategy is expected in small currencies with narrow markets.

\section{Conclusions}

We analyze market inefficiencies in the market for cryptocurrencies by providing a comprehensive analysis of short-term (over)reactions that follow significant price changes of such currencies. We consider a broad sample of the thirty cryptocurrencies with the highest market capitalization for an expanded timespan that includes the years 2015 to 2020 - or as long as the specific currency is available. We analyze the returns after the days with abnormal price changes and identify significant differences from normal returns using the $t$ test and the Mann-Whitney U test.

This kind of analysis has some tradition in the financial literature since similar research is provided for several other financial markets, while for cryptocurrencies, only a few papers exist that apply this methodological approach. We complement this literature in several important ways. We apply a different method to calculate the returns, i.e., end-of-the-day returns, which we apply together and compare to high-low returns that are used in the literature (see Caporale and Plastun, 2019). Both approaches have their pros and cons. The 
use of end-of-the-day returns seems more realistic with respect to trading strategies (since the daily high and low are never known in real time), and it enables us to consider negative and positive abnormal returns. However, we apply both approaches and compare their results.

In addition, we contribute by considering a much broader data sample that includes smaller currencies with lower market capitalization and trading volume. Thus, we can search for market size effects. By additionally using a broader set of thresholds (to identify abnormal returns), we can analyze whether the amount of the initial abnormal return is related to market inefficiencies, which is called a magnitude effect.

The results show that there is strong evidence for significant price patterns in the cryptomarket on postevent days. After positive abnormal price reactions, we observe positive returns on the first postevent day, $t+1$, that are significantly higher than the returns on "normal" days. These findings indicate underreactions of the market to the information that leads to the initially observed abnormal return. Additionally, after negative abnormal price changes, the postevent returns on the first day are significantly positive at all thresholds. This mean reversion indicates a strong overreaction on the negative information that leads to an initial abnormal drop in prices. It is interesting to see different patterns for negative and positive initial abnormal returns. However, both patterns are significant indicators of market inefficiencies.

Furthermore, a magnitude effect is observed: The magnitude of the initial abnormal return impacts the size of the market imperfection, i.e., how strong the market reacts on the following days. The higher the price change on event Day $t$ is, the higher the price change on the following Day $t+1$. In addition, the price reactions on postevent Days $t+2$ to $t+5$ were analyzed in this study. The results are somewhat mixed. A number of significant cases of tests of market movements on days $(t+2$ until $t+5)$ follow with higher lags of the event day. However, we do not observe significant price changes for all of the considered cases. Similar results are found when we consider averages calculated from cumulative returns for multiple days that follow on an event day, i.e., on short time periods. Overall, we can state that there is-at least weak-evidence for significant market reactions on later days.

In addition to multiple days of reactions after one abnormal return, we consider the case of multiple days of abnormal returns and the reactions thereafter; i.e., we analyze whether after a series of two and three consecutive days with an abnormal price change in the same direction, the return on the day following this series is significantly different from all normal returns. However, the results are mixed. For end-of-the-day returns, we found significant results for postevent Day $t+2$ after negative abnormal price changes and not in the other cases. For the high-low returns, most of the results are significant. By applying regression models, we provide additional evidence for both a market size and a magnitude effect. The market size effect is confirmed by significant dependencies of market inefficiencies on trading volume on the one hand and market capitalization of the currency on the other. The trading volume's impact is only significant for positive initial returns, whereas market capitalization is significant in both cases. The existence of a magnitude effect is confirmed by the regression models since we find a significant impact of the size of the initial abnormal return on the observed postevent returns. Thus, our results obtained with regression models confirm the findings that have been detected by the results for different thresholds, at least for negative abnormal price changes. As mentioned, different approaches exist to calculate the returns. The results discussed thus 
far rely on end-of-the-day returns, while other authors (e.g., Caporale and Plastun, 2019) use returns calculated by high and low prices. Both approaches have their pros and cons. For example, the use of end-of-the-day returns is related to a relatively simple trading strategy that could be easily implemented, which involves buying at the end of a day of an abnormal return-regardless of whether the abnormal return has been positive or negative (although for the latter, the effect is more pronounced)-and selling on the end of the next day. With more sophisticated research, one could approach the identification of high and low prices and exploit inefficiencies even more. To provide a comprehensive picture and robust results, we use both approaches. The results with the calculation method provided by Caporale and Plastun (2019) also point to significant overreaction patterns in the price history. As expected, the effects and the level of significance are mostly higher than for end-of-the-day returns.

To conclude, our paper shows that there is clear evidence of market inefficiencies in the market for cryptocurrencies regardless of whether we consider end-of-the-day returns or high-low returns. By tendency, the results for the latter are more significant. We detect overreactions in the case of negative abnormal returns and underreactions in the case of positive abnormal returns. Overall, there seems to be a stronger price reaction after negative abnormal price changes than after positive abnormal price changes, which coincides with the findings of earlier research. Furthermore, a clear magnitude effect is visible in our results, which has not yet been investigated in this way for the cryptomarket. We also find evidence for a market size effect indicated by a significant impact of trading volume as well as of market capitalization, which is another contribution to the existing literature. Overall, the results indicate a violation of the efficient market hypothesis in the cryptomarket in several ways. In addition, these empirical findings have important implications for the trading strategies of cryptoinvestors. With the help of a momentum strategy following positive abnormal price movements and a contrarian strategy following negative abnormal price changes, significant profits can be generated in the short term by exploiting these price patterns.

\section{References}

Aharon, D.Y., Qadan, M., 2018. Bitcoin and the day-of-the-week effect. Finance Research Letters. https://doi.org/10.1016/j.frl.2018.12.004

Arbel, A., Jaggi, B., 1982. Market Information Assimilation Related to Extreme Daily Price Jumps. Financial Analysts Journal 38, 60-66. https://doi.org/10.2469/faj.v38.n6.60

Atkins, A.B., Dyl, E.A., 1990. Price Reversals, Bid-Ask Spreads, and Market Efficiency. Journal of Financial and Quantitative Analysis 25, 535-547. https://doi.org/10.2307/2331015

Bariviera, A.F., Basgall, M.J., Hasperué, W., Naiouf, M., 2017. Some stylized facts of the Bitcoin market. Physica A: Statistical Mechanics and its Applications 484, 82-90. https://doi.org/10.1016/j.physa.2017.04.159

Borgards, O., Czudaj, R.L., 2020. The prevalence of price overreactions in the cryptocurrency market. Journal of International Financial Markets, Institutions and Money 65, 101194. https://doi.org/10.1016/j.intfin.2020.101194

Bouri, E., Gupta, R., Roubaud, D., 2018. Herding behaviour in cryptocurrencies. Finance Research Letters S1544612318303647. https://doi.org/10.1016/j.frl.2018.07.008 
Brown, K.C., Harlow, W.V., Tinic, S.M., 1988. Risk aversion, uncertain information, and market efficiency. Journal of Financial Economics 22, 355-385. https://doi.org/10.1016/0304405X(88)90075-X

Caporale, G.M., Plastun, A., 2020. Momentum effects in the cryptocurrency market after oneday abnormal returns. Financ Mark Portf Manag. https://doi.org/10.1007/s11408-020$00357-1$

Caporale, G.M., Plastun, A., 2019. Price overreactions in the cryptocurrency market. Journal of Economic Studies 46, 1137-1155. https://doi.org/10.1108/JES-09-2018-0310

Caporale, G.M., Plastun, A., 2018. The day of the week effect in the cryptocurrency market. Finance Research Letters. https://doi.org/10.1016/j.frl.2018.11.012

Chang, R.P., McLeavey, D.W., Rhee, S.G., 1995. Short-Term Abnormal Returns of the Contrarian Strategy in the Japanese Stock Market. Journal of Business Finance \& Accounting 22, 1035-1048. https://doi.org/10.1111/j.1468-5957.1995.tb00892.x

Chevapatrakul, T., Mascia, D.V., 2018. Detecting overreaction in the Bitcoin market: A quantile autoregression approach. Finance Research Letters S1544612318305920. https://doi.org/10.1016/j.frl.2018.11.004

Corbet, S., Katsiampa, P., 2018. Asymmetric mean reversion of Bitcoin price returns. International Review of Financial Analysis S1057521918306136. https://doi.org/10.1016/j.irfa.2018.10.004

Cox, D.R., Peterson, D.R., 1994. Stock Returns following Large One-Day Declines: Evidence on Short-Term Reversals and Longer-Term Performance. The Journal of Finance 49, 255-267. https://doi.org/10.1111/j.1540-6261.1994.tb04428.x

Daniel, K., Hirshleifer, D., Subrahmanyam, A., 1998. Investor Psychology and Security Market Under- and Overreactions. The Journal of Finance 53, 1839-1885. https://doi.org/10.1111/0022-1082.00077

De Bondt, W.F.M., Thaler, R., 1985. Does the Stock Market Overreact? The Journal of Finance 40, 793-805. https://doi.org/10.1111/j.1540-6261.1985.tb05004.x

Décourt, R.F., Chohan, U.W., Perugini, M.L., 2017. Bitcoin returns and the monday effect. Horizontes Empresariales 16.

Karalevicius, V., Degrande, N., De Weerdt, J., 2017. Using sentiment analysis to predict interday Bitcoin price movements. The Journal of Risk Finance 19, 56-75. https://doi.org/10.1108/JRF-06-2017-0092

Kosc, K., Sakowski, P., Ślepaczuk, R., 2019. Momentum and contrarian effects on the cryptocurrency market. Physica A: Statistical Mechanics and its Applications 523, 691-701. https://doi.org/10.1016/j.physa.2019.02.057

Kurihara, Y., Fukushima, A., 2017. The Market Efficiency of Bitcoin: A Weekly Anomaly Perspective. Journal of Applied Finance \& Banking 7, 1-4.

Larson, S.J., Madura, J., 2003. What Drives Stock Price Behavior Following Extreme One-Day Returns. Journal of Financial Research 26, 113-127. https://doi.org/10.1111/14756803.00048

Latif, S.R., Mohd, M.A., Mohd Amin, M.N., Mohamad, A.I., 2017. Testing the weak form of efficient market in cryptocurrency. Journal of Engineering and Applied Sciences 12, 22852288. https://doi.org/10.3923/jeasci.2017.2285.2288

Lobe, S., Rieks, J., 2011. Short-term market overreaction on the Frankfurt stock exchange. The Quarterly Review of Economics and Finance 51, 113-123. https://doi.org/10.1016/j.qref.2010.12.002 
Ma, Y., Tang, A.P., Hasan, T., 2005. The Stock Price Overreaction Effect: Evidence on Nasdaq Stocks. Quarterly Journal of Business and Economics 44, 113-127.

Murray Leclair, E., 2018. Herding in the cryptocurrency market. https://doi.org/10.13140/RG.2.2.26154.11204

Nadarajah, S., Chu, J., 2017. On the inefficiency of Bitcoin. Economics Letters 150, 6-9. https://doi.org/10.1016/j.econlet.2016.10.033

Poyser, 0., 2018. Herding behavior in cryptocurrency markets (No. 1806.11348), Papers. arXiv.org.

Spyrou, S., Kassimatis, K., Galariotis, E., 2007. Short-term overreaction, underreaction and efficient reaction: Evidence from the London Stock Exchange. Applied Financial Economics 17, 221-235. https://doi.org/10.1080/09603100600639868

Urquhart, A., 2016. The inefficiency of Bitcoin. Economics Letters 148, 80-82. https://doi.org/10.1016/j.econlet.2016.09.019 


\section{Appendix}

Table 1: Considered coins

\begin{tabular}{|c|c|c|c|c|c|}
\hline coin & USD.price & USD.market.cap & start.date & mean.return & sd.return \\
\hline Bitcoin & 29001.72 & 539.05 & 01.01 .2015 & 0.21 & 3.91 \\
\hline Ethereum & 737.80 & 84.16 & 08.08 .2015 & 0.35 & 6.16 \\
\hline Tether & 1.00 & 20.94 & 26.02.2015 & -0.01 & 1.92 \\
\hline XRP & 0.22 & 9.98 & 01.01 .2015 & 0.10 & 6.56 \\
\hline Litecoin & 124.69 & 8.26 & 01.01 .2015 & 0.17 & 5.71 \\
\hline Bitcoin Cash & 343.05 & 6.38 & 24.07.2017 & -0.02 & 7.36 \\
\hline Cardano & 0.18 & 5.64 & 02.10 .2017 & 0.16 & 7.36 \\
\hline Binance Coin & 37.38 & 5.40 & 26.07.2017 & 0.47 & 7.17 \\
\hline Chainlink & 11.27 & 4.49 & 21.09.2017 & 0.35 & 7.75 \\
\hline USD Coin & 1.00 & 3.90 & 09.10 .2018 & 0.00 & 0.51 \\
\hline Wrapped Bitcoin & 28977.74 & 3.35 & 31.01 .2019 & 0.30 & 4.19 \\
\hline Bitcoin SV & 163.63 & 3.05 & 10.11.2018 & 0.08 & 8.46 \\
\hline Stellar & 0.13 & 2.81 & 01.01 .2015 & 0.14 & 7.14 \\
\hline Monero & 156.57 & 2.79 & 01.01 .2015 & 0.27 & 6.33 \\
\hline EOS & 2.60 & 2.44 & 02.07.2017 & 0.00 & 7.03 \\
\hline TRON & 0.03 & 1.92 & 14.09.2017 & 0.24 & 8.53 \\
\hline THETA & 1.86 & 1.86 & 18.01.2018 & 0.21 & 7.75 \\
\hline NEM & 0.20 & 1.84 & 02.04 .2015 & 0.31 & 7.75 \\
\hline Tezos & 2.02 & 1.52 & 03.10 .2017 & 0.00 & 7.15 \\
\hline UNUS SED LEO & 1.36 & 1.36 & 22.05.2019 & 0.03 & 2.13 \\
\hline Cosmos & 6.49 & 1.35 & 15.03.2019 & -0.02 & 6.80 \\
\hline Crypto.com Coin & 0.06 & 1.31 & 15.12.2018 & 0.16 & 6.85 \\
\hline Celsius & 5.46 & 1.30 & 02.10 .2018 & 0.55 & 7.50 \\
\hline VeChain & 0.02 & 1.21 & 04.08 .2018 & 0.04 & 6.44 \\
\hline Dai & 1.00 & 1.17 & 23.11.2019 & 0.00 & 1.20 \\
\hline Filecoin & 24.35 & 1.09 & 14.12.2017 & 0.07 & 10.91 \\
\hline
\end{tabular}




\begin{tabular}{|c|c|c|c|c|c|}
\hline coin & USD.price & USD.market.cap & start.date & mean.return & sd.return \\
\hline $\mathrm{NeO}$ & 14.32 & 1.01 & 10.09 .2016 & 0.23 & 8.28 \\
\hline Revain & 0.01 & 0.99 & 02.11 .2017 & -0.16 & 7.14 \\
\hline Dash & 99.61 & 0.99 & 01.01.2015 & 0.18 & 5.76 \\
\hline Binance USD & 1.00 & 0.98 & 21.09.2019 & 0.00 & 0.55 \\
\hline Zilliqa & 0.08 & 0.89 & 26.01 .2018 & -0.03 & 6.91 \\
\hline Huobi Token & 4.37 & 0.87 & 04.02 .2018 & 0.11 & 4.84 \\
\hline IOTA & 0.30 & 0.83 & 14.06.2017 & -0.04 & 7.07 \\
\hline Synthetix & 7.22 & 0.80 & 15.03 .2018 & 0.28 & 8.52 \\
\hline Zcash & 63.99 & 0.69 & 30.10 .2016 & -0.14 & 7.22 \\
\hline Ethereum Classic & 5.68 & 0.66 & 25.07 .2016 & 0.14 & 7.39 \\
\hline Waves & 6.20 & 0.64 & 03.06.2016 & 0.10 & 7.38 \\
\hline Kusama & 71.96 & 0.61 & 13.12.2019 & 0.97 & 9.17 \\
\hline Dogecoin & 0.00 & 0.60 & 01.01 .2015 & 0.15 & 5.91 \\
\hline Maker & 586.96 & 0.59 & 30.01 .2017 & 0.28 & 9.67 \\
\hline FTX Token & 5.77 & 0.54 & 01.08 .2019 & 0.23 & 3.97 \\
\hline Decred & 40.78 & 0.51 & 11.02 .2016 & 0.20 & 7.11 \\
\hline OKB & 7.78 & 0.47 & 01.05 .2019 & 0.26 & 4.84 \\
\hline UMA & 7.64 & 0.42 & 26.05 .2020 & 0.84 & 9.71 \\
\hline Algorand & 0.33 & 0.40 & 22.06.2019 & -0.33 & 7.25 \\
\hline Ontology & 0.44 & 0.35 & 09.03 .2018 & -0.13 & 6.74 \\
\hline DigiByte & 0.02 & 0.35 & 01.01 .2015 & 0.29 & 9.10 \\
\hline OMG Network & 2.45 & 0.34 & 15.07.2017 & 0.14 & 7.75 \\
\hline Terra & 0.65 & 0.32 & 27.07.2019 & -0.13 & 5.96 \\
\hline Nexo & 0.55 & 0.31 & 02.05 .2018 & 0.10 & 6.86 \\
\hline
\end{tabular}

Price and market capitalization on 31.12.2020

Market capitalization in billion US-Dollar

mean.return and sd.return represent the average and the standard deviation of all daily returns over the entire period 
Table 2: Test results: positive abormal price changes

\begin{tabular}{|c|c|c|c|c|c|c|c|}
\hline & & & & & & t.test & wilcox.test \\
\hline threshold & obs.cR & obs.nR & event.prob* & mean.cR & mean.nR & p.value.t & p.value.w \\
\hline 1.0 & 5,692 & 56,608 & 9.14 & 0.30 & 0.13 & 0.225 & $0.000 * * *$ \\
\hline 1.5 & 2,930 & 59,370 & 4.70 & 0.40 & 0.13 & 0.241 & $0.000 * * *$ \\
\hline 2.0 & 1,653 & 60,647 & 2.65 & 0.50 & 0.14 & 0.295 & $0.000 * * *$ \\
\hline 2.5 & 987 & 61,313 & 1.58 & 0.67 & 0.14 & 0.304 & $0.000 * * *$ \\
\hline 3.0 & 608 & 61,692 & 0.98 & 0.61 & 0.14 & 0.518 & $0.002 * * *$ \\
\hline 3.5 & 396 & 61,904 & 0.64 & 1.08 & 0.14 & 0.355 & $0.087^{*}$ \\
\hline 4.0 & 271 & 62,029 & 0.43 & 0.37 & 0.15 & 0.863 & 0.015 * * \\
\hline 4.5 & 196 & 62,104 & 0.31 & -1.12 & 0.15 & 0.379 & $0.003 * * *$ \\
\hline 5.0 & 139 & 62,161 & 0.22 & -2.20 & 0.15 & 0.193 & $0.001 * * *$ \\
\hline 5.5 & 106 & 62,194 & 0.17 & -1.52 & 0.15 & 0.429 & 0.038 * * \\
\hline 6.0 & 81 & 62,219 & 0.13 & -1.60 & 0.15 & 0.498 & 0.076 * \\
\hline
\end{tabular}

obs.cR and obs.nR represent the number of obervations of $c R$-returns and nR-returns mean.cR and mean.nR represent the mean of all $c R$ - and $n R$-returns

Significance levels: ${ }^{* *}=0.01,{ }^{* *}=0.05,{ }^{*}=0.1$ (two-tailed test)

* event probability in percent 
Table 3: Test results: negative abormal price changes

\begin{tabular}{rrrrrrrr}
\hline threshold & obs.cR & obs.nR & event.prob* & mean.cR & mean.nR & p.value.t & p.value.w \\
\hline 1.0 & 5,613 & 56,687 & 9.01 & 0.90 & 0.07 & $0.000 * * *$ & $0.000 * * *$ \\
1.5 & 2,663 & 59,637 & 4.27 & 1.68 & 0.08 & $0.000 * * *$ & $0.000 * * *$ \\
2.0 & 1,316 & 60,984 & 2.11 & 2.27 & 0.10 & $0.000 * * *$ & $0.000 * * *$ \\
2.5 & 701 & 61,599 & 1.13 & 2.97 & 0.12 & $0.000 * * *$ & $0.000 * * *$ \\
3.0 & 388 & 61,912 & 0.62 & 4.36 & 0.12 & $0.000 * * *$ & $0.000 * * *$ \\
3.5 & 236 & 62,064 & 0.38 & 5.79 & 0.13 & $0.000 * * *$ & $0.000 * * *$ \\
4.0 & 151 & 62,149 & 0.24 & 6.39 & 0.13 & $0.000 * * *$ & $0.000 * * *$ \\
4.5 & 110 & 62,190 & 0.18 & 7.75 & 0.13 & $0.000 * * *$ & $0.000 * * *$ \\
5.0 & 84 & 62,216 & 0.13 & 9.21 & 0.14 & $0.000 * * *$ & $0.000 * * *$ \\
5.5 & 67 & 62,233 & 0.11 & 9.55 & 0.14 & $0.000 * * *$ & $0.000 * * *$ \\
6.0 & 54 & 62,246 & 0.09 & 10.41 & 0.14 & $0.000 * * *$ & $0.000 * * *$
\end{tabular}


Postevent returns after positive abnormal price changes for the following 5 days for different thresholds

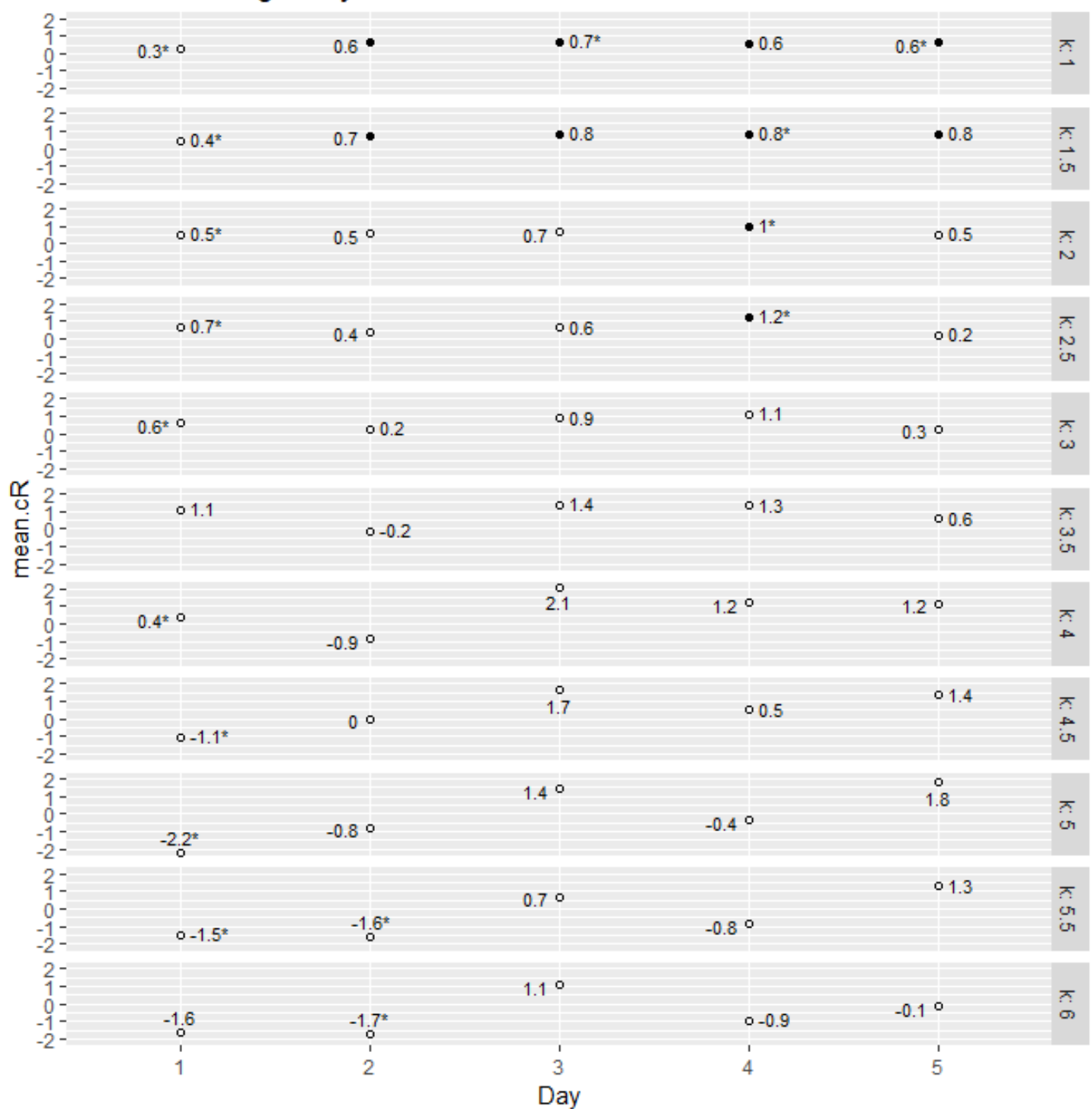

significant values $(p<0.05)$ according to the t-test are displayed as filled circles, insignificant as empty circles significant values $(p<0.05)$ according to the Wilcoxon-test are marked with a asterisk the numbers on the right side represent the thresholds

Figure 1: Postevent returns after positive abnormal price changes 
Postevent returns after negative abnormal price changes for the following 5 days for different thresholds

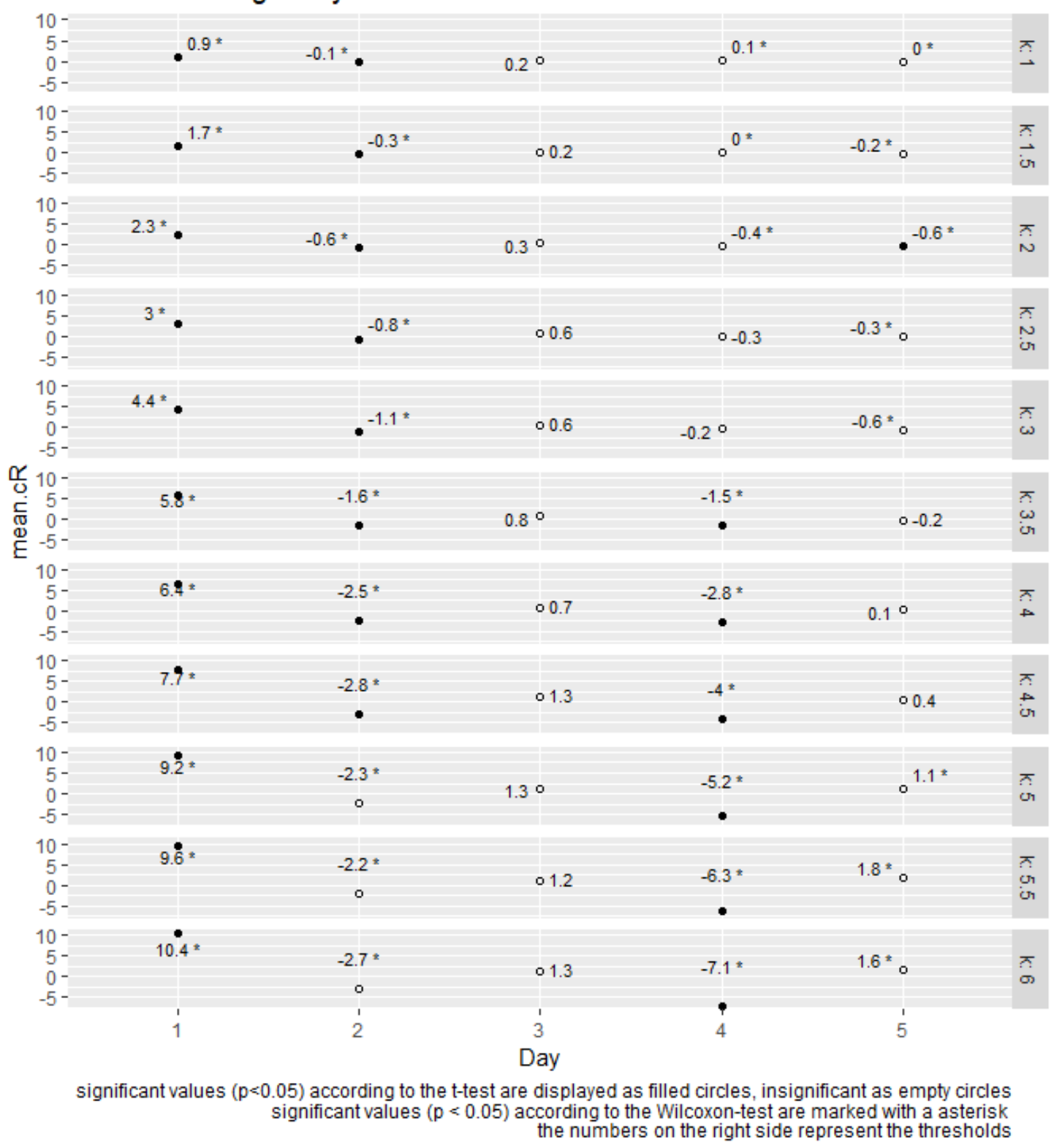

Figure 2: Postevent returns after negative abnormal price changes 
Sum of the postevent returns over several days after positive abnormal price changes

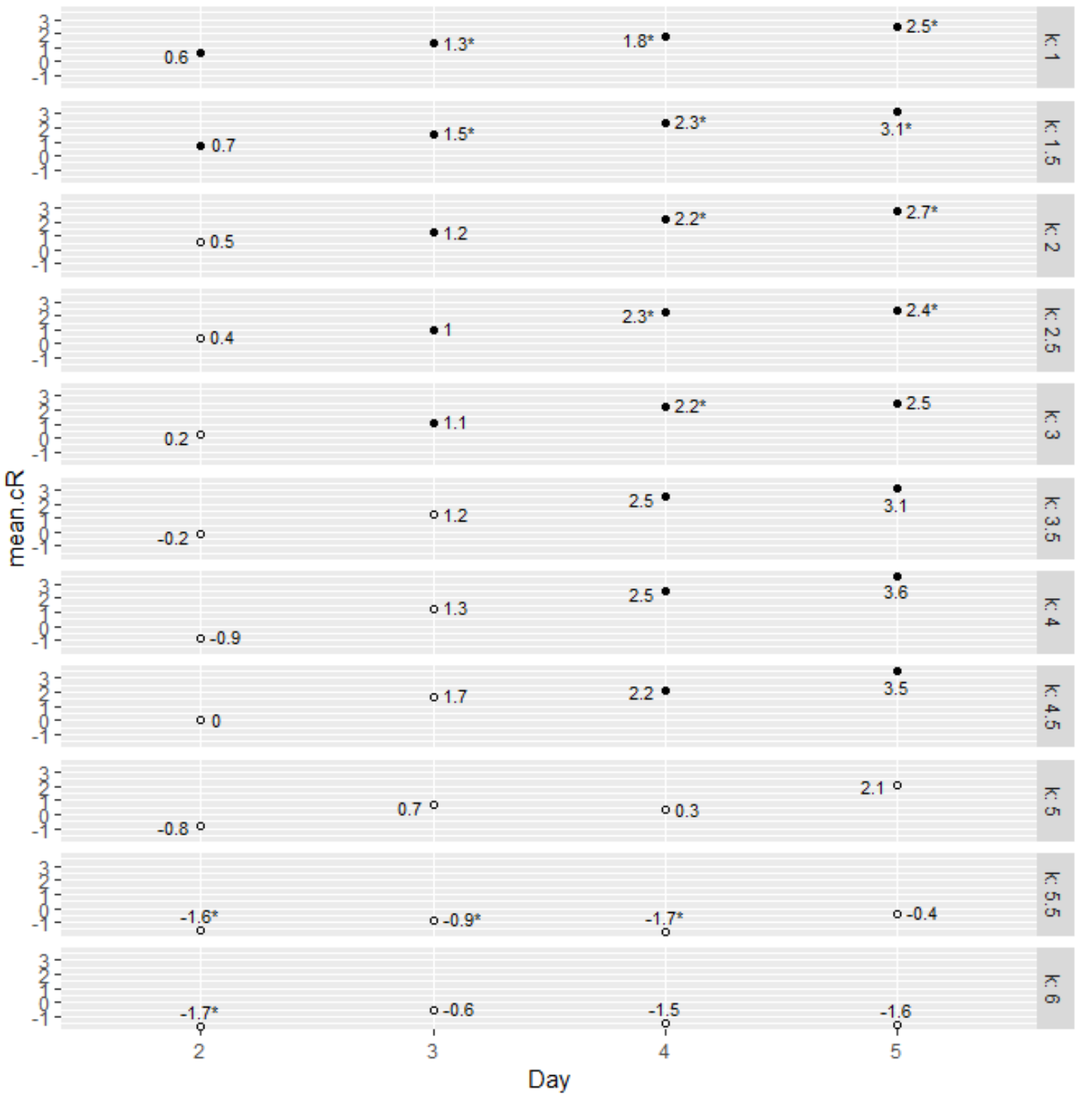

significant values $(p<0.05)$ according to the t-test are displayed as filled circles, insignificant as empty circles significant values $(p<0.05)$ according to the Wilcoxon-test are marked with a asterisk the numbers on the right side represent the thresholds

Figure 3: Sum of the postevent returns over several days after positive abnormal price changes 
Sum of the postevent returns over several days after negative abnormal price changes

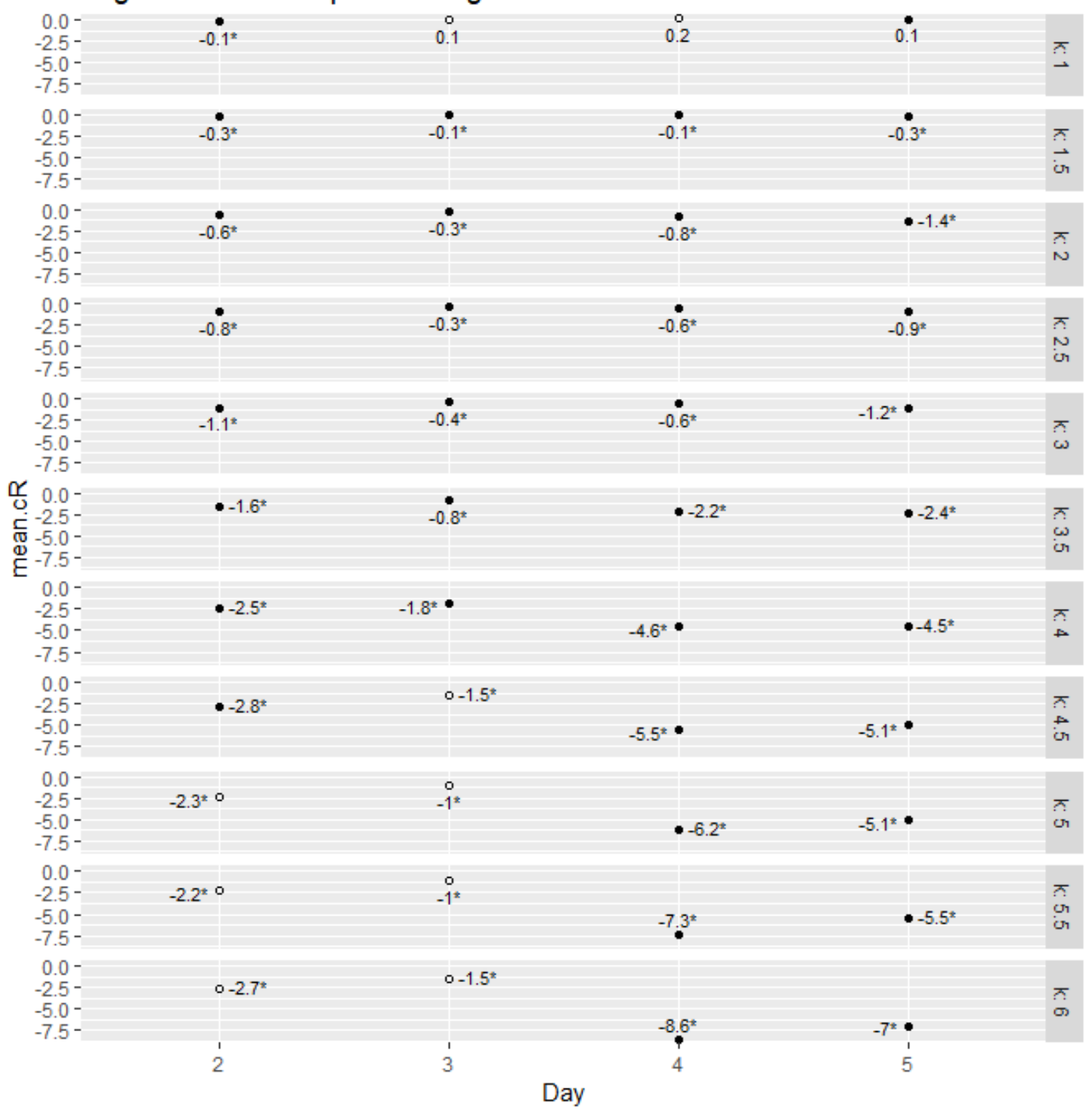

significant values ( $<<0.05)$ according to the t-test are displayed as filled circles, insignificant as empty circles significant values $(p<0.05)$ according to the Wilcoxon-test are marked with a asterisk the numbers on the right side represent the thresholds

Figure 4: Sum of the postevent returns over several days after positive abnormal price changes 
Table 4: Test results: 2 and 3 consecutive abnormal price changes

\begin{tabular}{|c|c|c|c|c|c|c|}
\hline & & & & & t.test & wilcox.test \\
\hline consec.days & obs.cR & obs.nR & mean.cR & mean.nR & p.value.t & p.value.w \\
\hline \multicolumn{7}{|l|}{ positive abnormal price changes } \\
\hline 2 & 779 & 61,571 & 0.11 & 0.15 & 0.936 & $0.000 * * *$ \\
\hline 3 & 162 & 62,188 & 1.72 & 0.15 & 0.234 & 0.316 \\
\hline \multicolumn{7}{|l|}{ negative abnormal price changes } \\
\hline 2 & 680 & 61,670 & 2.62 & 0.12 & $0.000 * * *$ & $0.000 * * *$ \\
\hline 3 & 75 & 62,275 & 1.70 & 0.15 & 0.234 & $0.004 * * *$ \\
\hline
\end{tabular}

'consec.days' shows the number of consecutive days with an abnormal price change in the same direction

Only the threshold $\mathrm{k}=1$ is used in this calculation

Table 5: Univariate OLS-regressions for positive abnormal price changes

\begin{tabular}{lllll}
\hline variable & estimate & std.error & statistic & p.value \\
\hline aR & 0.08 & 0.10 & 0.87 & 0.38 \\
MA_volume & -0.07 & 0.02 & -3.42 & $0.00 * * *$ \\
MA_market_cap & -0.01 & 0.00 & -2.36 & $0.02 * *$ \\
\hline
\end{tabular}

Univariate OLS-regressions with robust standard errors (HC3)

Dependent variable: $\mathrm{cR}$ (return on the postevent Day $\mathrm{t}+1$ )

$\mathrm{aR}=$ abnormal return on the event Day $\mathrm{t}=0$

MA_Volume $=$ Moving average of the trading volume

MA_market_cap $=$ Moving average of the market capitalization

Significance levels: ${ }^{* *}=0.01,{ }^{* *}=0.05,{ }^{*}=0.1$ 
Table 6: Univariate OLS-regressions for negative abnormal price changes

\begin{tabular}{lllll}
\hline variable & estimate & std.error & statistic & p.value \\
\hline aR & -0.19 & 0.02 & -8.64 & $0.00 * * *$ \\
MA_volume & -0.02 & 0.02 & -1.19 & 0.24 \\
MA_market_cap & -0.01 & 0.00 & -2.88 & $0.00 * * *$ \\
\hline
\end{tabular}

Univariate OLS-regressions with robust standard errors (HC3)

Dependent variable: $c R$ (return on the postevent Day $t+1$ )

$\mathrm{aR}=$ abnormal return on the event Day $\mathrm{t}=0$

MA_Volume $=$ Moving average of the trading volume

MA_market_cap $=$ Moving average of the market capitalization

Significance levels: ${ }^{* *}=0.01,{ }^{* *}=0.05,{ }^{*}=0.1$ 
Table 7: Test results: positive abormal price changes

\begin{tabular}{|c|c|c|c|c|c|c|c|}
\hline & & & & & & t.test & wilcox.test \\
\hline threshold & obs.cR & obs.nR & event.prob* & mean.cR & mean.nR & p.value.t & p.value.w \\
\hline 1.0 & 6,510 & 55,840 & 10.44 & 8.55 & 7.29 & $0.000 * * *$ & 0.296 \\
\hline 1.5 & 3,845 & 58,505 & 6.17 & 9.60 & 7.58 & $0.000 * * *$ & $0.000 * * *$ \\
\hline 2.0 & 2,395 & 59,955 & 3.84 & 10.56 & 7.77 & $0.000 * * *$ & $0.000 * * *$ \\
\hline 2.5 & 1,622 & 60,728 & 2.60 & 11.50 & 7.89 & $0.000 * * *$ & $0.000 * * *$ \\
\hline 3.0 & 1,113 & 61,237 & 1.79 & 12.66 & 7.98 & $0.000 * * *$ & $0.000 * * *$ \\
\hline 3.5 & 779 & 61,571 & 1.25 & 13.39 & 8.05 & $0.000 * * *$ & $0.000 * * *$ \\
\hline 4.0 & 577 & 61,773 & 0.93 & 14.18 & 8.10 & $0.000 * * *$ & $0.000 * * *$ \\
\hline 4.5 & 430 & 61,920 & 0.69 & 14.71 & 8.14 & $0.000 * * *$ & $0.000 * * *$ \\
\hline 5.0 & 326 & 62,024 & 0.52 & 15.71 & 8.17 & $0.000 * * *$ & $0.000 * * *$ \\
\hline 5.5 & 243 & 62,107 & 0.39 & 16.33 & 8.20 & $0.000 * * *$ & $0.000 * * *$ \\
\hline 6.0 & 186 & 62,164 & 0.30 & 16.52 & 8.22 & $0.000 * * *$ & $0.000 * * *$ \\
\hline
\end{tabular}

obs.cR and obs.nR represent the number of obervations of $c R$-returns and nR-returns

mean.cR and mean.nR represent the mean of all $c R$ - and $n R$-returns

Significance levels: ${ }^{* * *}=0.001,{ }^{* *}=0.01,{ }^{*}=0.05$ (two-tailed test)

* event probability in percent 
Postevent returns after positive abnormal price changes

for the following 5 days for different thresholds

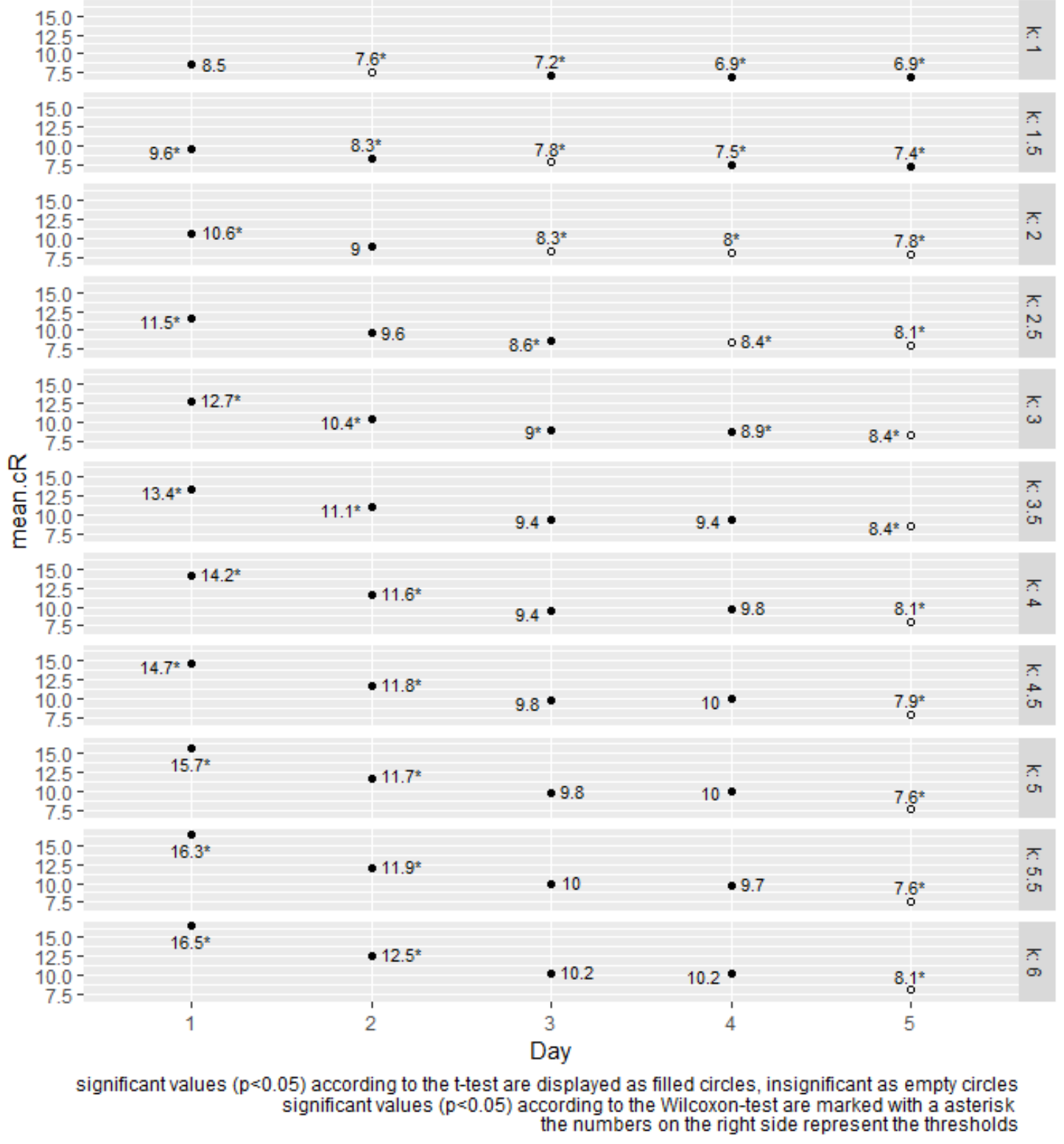

Figure 5: Postevent returns after positive abnormal price changes 
Table 8: Test results: 2 and 3 consecutive abnormal price changes

\begin{tabular}{rrrrrrr}
\hline & & & \multicolumn{1}{c}{ t.test } & wilcox.test \\
\hline consec.days & obs.cR & obs.nR & mean.cR & mean.nR & p.value.t & p.value.w \\
\hline 2 & 1,345 & 61,055 & 8.59 & 8.11 & $0.069 *$ & $0.004 * * *$ \\
3 & 562 & 61,838 & 10.61 & 8.21 & $0.000 * * *$ & $0.000 * * *$ \\
\hline
\end{tabular}

'consec.days' shows the number of consecutive days with an abnormal price change in the same direction

Only the threshold with one standard deviation is used in this calculation

Table 9: Univariate OLS-regressions for positive abnormal price changes

\begin{tabular}{lrrrr}
\hline variable & estimate & std.error & statistic & p.value \\
\hline aR & 0.23 & 0.02 & 12.21 & $0 * * *$ \\
MA_volume & -0.14 & 0.01 & -9.68 & $0 * * *$ \\
MA_market_cap & -0.01 & 0.00 & -3.10 & $0 * * *$ \\
\hline
\end{tabular}

Univariate OLS-regressions with robust standard errors (HC3)

variable: $c R$ (return on the postevent Day $t+1$ )

$\mathrm{aR}=$ abnormal return on the event Day $\mathrm{t}=0$

MA_Volume $=$ Moving average of the trading volume

MA_market_cap $=$ Moving average of the market capitalization

Significance levels: ${ }^{* *}=0.01,{ }^{* *}=0.05,{ }^{*}=0.1$ 